\title{
Experimental Demonstration of Effective Medium Approximation Breakdown in Deeply Subwavelength All-Dielectric Multilayers
}

Zhukovsky, Sergei; Andryieuski, Andrei; Takayama, Osamu; Shkondin, Evgeniy; Malureanu, Radu; Jensen, Flemming; Lavrinenko, Andrei

Published in:

Physical Review Letters

Link to article, DOI:

10.1103/physrevlett.115.177402

Publication date:

2015

Document Version

Publisher's PDF, also known as Version of record

Link back to DTU Orbit

Citation (APA):

Zhukovsky, S., Andryieuski, A., Takayama, O., Shkondin, E., Malureanu, R., Jensen, F., \& Lavrinenko, A. (2015). Experimental Demonstration of Effective Medium Approximation Breakdown in Deeply Subwavelength All-Dielectric Multilayers. Physical Review Letters, 115(17), 177402.

https://doi.org/10.1103/physrevlett.115.177402

\section{General rights}

Copyright and moral rights for the publications made accessible in the public portal are retained by the authors and/or other copyright owners and it is a condition of accessing publications that users recognise and abide by the legal requirements associated with these rights.

- Users may download and print one copy of any publication from the public portal for the purpose of private study or research.

- You may not further distribute the material or use it for any profit-making activity or commercial gain

- You may freely distribute the URL identifying the publication in the public portal 


\title{
Experimental Demonstration of Effective Medium Approximation Breakdown in Deeply Subwavelength All-Dielectric Multilayers
}

\author{
Sergei V. Zhukovsky, ${ }^{1,2, *}$ Andrei Andryieuski, ${ }^{1}$ Osamu Takayama, ${ }^{1}$ Evgeniy Shkondin, ${ }^{1,3}$ Radu Malureanu, ${ }^{1}$ \\ Flemming Jensen, ${ }^{3}$ and Andrei V. Lavrinenko ${ }^{1, \dagger}$ \\ ${ }^{1}$ DTU Fotonik-Department of Photonics Engineering, Technical University of Denmark, \\ Ørsteds Plads 343, DK-2800 Kgs. Lyngby, Denmark \\ ${ }^{2}$ ITMO University, Kronverksky pr. 49, St. Petersburg, 197101, Russia \\ ${ }^{3}$ DTU Danchip - National Center for Micro- and Nanofabrication, Technical University of Denmark, \\ Ørsteds Plads 347, DK-2800 Kgs. Lyngby, Denmark \\ (Received 22 June 2015; published 23 October 2015)
}

\begin{abstract}
We report the first experimental demonstration of anomalous breakdown of the effective medium approximation in all-dielectric deeply subwavelength thickness $(d \sim \lambda / 160-\lambda / 30)$ multilayers, as recently predicted theoretically [H. H. Sheinfux et al., Phys. Rev. Lett. 113, 243901 (2014)]. Multilayer stacks are composed of alternating alumina and titania layers fabricated using atomic layer deposition. For light incident on such multilayers at angles near the total internal reflection, we observe pronounced differences in the reflectance spectra for structures with 10- vs 20-nm thick layers, as well as for structures with different layers ordering, contrary to the predictions of the effective medium approximation. The reflectance difference can reach values up to 0.5 , owing to the chosen geometrical configuration with an additional resonator layer employed for the enhancement of the effect. Our results are important for the development of new high-precision multilayer ellipsometry methods and schemes, as well as in a broad range of sensing applications.
\end{abstract}

PACS numbers: 78.67.Pt, 42.25.Gy, 78.20.-e, 81.15.Gh

Photonic multilayers are one of the most widely studied systems in the broader topic of optics of inhomogeneous media [1-4]. Most optical effects of such multilayers arise from interference effects underlying the photonic band gap phenomena [5], and therefore are traditionally associated with multilayers, where layer thickness $d$ is comparable to the wavelength of light $\lambda$. For example, the well-known Bragg mirror comprising a stack of alternating low- and high-index dielectric layers exhibits maximum reflectance if the optical thickness of each layer is close to $\lambda / 4$ [6].

From this point of view, the case of multilayers with much thinner layers with thicknesses $d \ll \lambda$ was traditionally regarded as nearly trivial. Indeed, in such a "deeply subwavelength" structure the field variation inside a single layer should be very small, leading to negligibly weak interference effects. Therefore, one used to assume that a light wave interacts with the structure as a whole rather than with its individual layers. The structure can thus be treated as a piece of homogeneous uniaxial material characterized by effective parameters [7]. The applicability of this homogenization approach to all-dielectric multilayers with ultrathin layers $d \ll \lambda$ has always been undoubted, in much the same way as ordinary materials are treated as homogeneous media despite having atomic, molecular, or any other intrinsic structure.

However, a recent paper by Sheinfux et al. [8] showed theoretically that this commonly believed assumption may fail in certain circumstances. Namely, when light is incident on a multilayer at an angle close to that of the total internal reflection (TIR), the actual multilayer and its effectivemedium approximation (EMA) model can have significantly different transmission spectra despite the layer thicknesses smaller than $\lambda / 50$. Moreover, it was shown that the spectra become sensitive to variations of $d$ on the scale of $1 \mathrm{~nm}$ (i.e., $<\lambda / 500$ ), as well as on the layer ordering (i.e., the multilayer $H L H L \ldots H L$ vs $L H L H \ldots L H$, where $H$ and $L$ stand for high- and lowindex layers). Both effects are totally contrary to the predictions of the EMA, which is independent of individual layer features. Physically, the EMA breaks down close to the TIR angle because the waves become evanescent in low-index layers but remain propagating in the high-index layers. Since the layers are deeply subwavelength, the impinging wave may still propagate through the multilayer via tunneling, whereas the EMA does not capture this physics and prohibits wave propagation. In other words, the wave propagating through the multilayer accumulates its phase via repeated Fresnel reflection at layer interfaces rather than via propagation through the bulk of the layers, thereby causing EMA to fail [8].

This anomalous EMA breakdown is both enlightening and practically promising (e.g., for sensing and switching applications); therefore, it would be of great importance to observe this effect experimentally. However, the effect reported in Ref. [8] only becomes noticeable in multilayers containing at least a few hundreds of 10 -nm thick layers 
with $<1 \mathrm{~nm}$ thickness tolerance, which is extremely challenging to fabricate.

In this Letter, we report the experimental observation of the EMA breakdown effect by placing subwavelength multilayers on a photonic resonator. The resonator greatly improves the sensitivity of the scheme towards tiny impedance mismatches at the output interface of the multilayer [9]. This makes the EMA breakdown measurable for structures containing only a few tens of layers, significantly relaxing the fabrication requirements. The multilayers themselves were fabricated using atomic layer deposition (ALD), chosen for its several advantages compared to other deposition techniques (e.g., precise thickness control, excellent step coverage, and conformal deposition [10]). Our measurements confirm that the reflection spectra of the subwavelength multilayers become sensitive to both layer thicknesses and layer ordering in a range of incident angles immediately preceding the TIR angle, in full agreement with the theoretical predictions.

The layout of the experimental arrangement is shown in Fig. 1(a). The central element of the setup is a subwavelength multilayer film with total thickness $200 \mathrm{~nm}$, containing alternating low-index $(L)$ layers made of alumina $\left(\mathrm{Al}_{2} \mathrm{O}_{3}\right)$ and high-index $(H)$ layers made of titania $\left(\mathrm{TiO}_{2}\right)$. The layer of silicon nitride $\left(\mathrm{Si}_{3} \mathrm{~N}_{4}\right)$ beneath the multilayer serves as an index matched $\left(n_{\mathrm{SiN}} \simeq \sqrt{\left(n_{H}^{2}+n_{L}^{2}\right) / 2}\right)$ resonator layer. Such photonic resonators are commonly used as a tool to enhance all effects related to impedance matching, which is known to be important in the EMA breakdown physics [8]. In our case, the resonator makes the
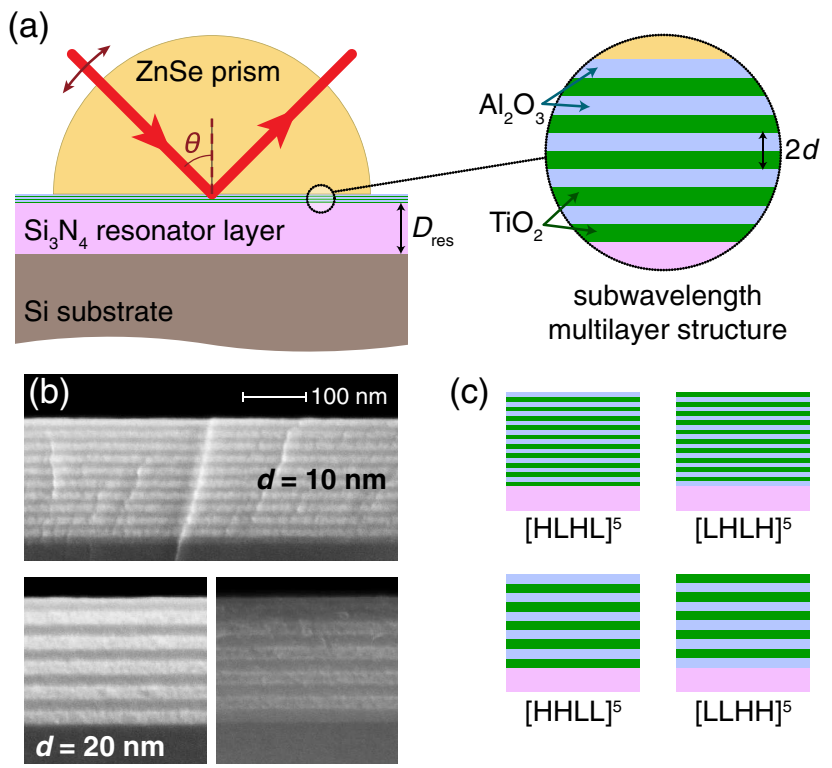

(c)

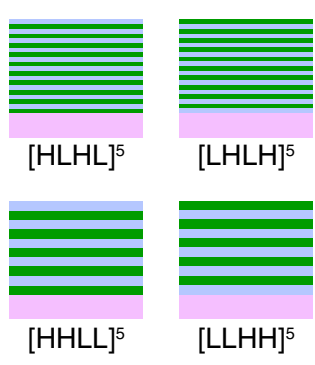

FIG. 1 (color online). (a) Layout of the experimental setup. (b) SEM images of the cross section of ALD-fabricated multilayers with layer thicknesses 10 and $20 \mathrm{~nm}$ (with different layer ordering). (c) Schematics of the four fabricated samples differing by the layer thicknesses and ordering. reflectance of the entire structure extremely sensitive to the slight impedance mismatch at the multilayer-resonator boundary [9], which is necessary to observe the EMA breakdown. Since the incident angles of interest have to be near-TIR, a high-index ambient medium is required. For this purpose a semicylindrical prism made of zinc selenide $\left(n_{\mathrm{ZnSe}}>n_{H}>n_{L}\right)$ was used.

All the samples were prepared and assembled in a class 100 clean room. The fabrication of the multilayers was performed in a hot-wall ALD system (Picosun R200). The precursors used for $\mathrm{Al}_{2} \mathrm{O}_{3}$ and $\mathrm{TiO}_{2}$ deposition were trimethylaluminum $\mathrm{Al}\left(\mathrm{CH}_{3}\right)_{3}$ and titanium tetrachloride $\mathrm{TiCl}_{4}$, respectively (both from Sigma-Aldrich). In both processes the oxidant source was deionized water. The deposition temperature was $120^{\circ} \mathrm{C}$ in order to prevent the crystal anatase phase transition of $\mathrm{TiO}_{2}$ known to occur at temperatures above $150^{\circ} \mathrm{C}$ [11], which increases the films' roughness. The growth rates of $\mathrm{Al}_{2} \mathrm{O}_{3}$ and $\mathrm{TiO}_{2}$ films were determined to be 0.047 and $0.089 \mathrm{~nm} /$ cycle, respectively (in agreement with previously reported data [12]), using varying-time deposition with ellipsometric characterization of the films' thicknesses and refractive indices (VASE, J.A. Woollam Co.).

The index matched resonator layer was fabricated by

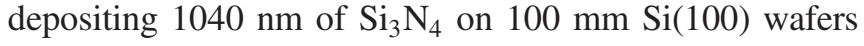
using low-pressure chemical vapor deposition. The process was carried out at $780^{\circ} \mathrm{C}$ with ammonia $\left(\mathrm{NH}_{3}\right)$ and dichlorsilane $\left(\mathrm{SiH}_{2} \mathrm{Cl}_{2}\right)$ as reactive gases. The thickness and refractive index of the deposited $\mathrm{Si}_{3} \mathrm{~N}_{4}$ film were tested using spectroscopic ellipsometry. The film was carefully inspected for cracks, particles, and other defects using dark field optical microscopy. The wafer with the best-quality $\mathrm{Si}_{3} \mathrm{~N}_{4}$ coating was selected and cleaved in pieces, which were used as substrates for the subsequent deposition of $\mathrm{Al}_{2} \mathrm{O}_{3} / \mathrm{TiO}_{2}$ multilayers. Before inserting each substrate into the ALD reactor, it was placed on a $\mathrm{Si}$ carrier wafer. Therefore the $\mathrm{Al}_{2} \mathrm{O}_{3} / \mathrm{TiO}_{2}$ multilayers were grown not only on the $\mathrm{Si}_{3} \mathrm{~N}_{4}$ layer but also on the dummy carrier wafer. After the ALD process, the dummy wafer was cleaved, and its cross section was characterized using scanning electron microscopy (SEM). The SEM images reveal high-quality homogeneous, conformal coatings, as seen in Fig. 1(b).

We fabricated four different configurations of multilayer arrangement, shown schematically in Fig. 1(c). Two of them comprise 20 alternating $\mathrm{TiO}_{2}$ and $\mathrm{Al}_{2} \mathrm{O}_{3}$ layers with $d=10 \mathrm{~nm}$ thickness and different layer ordering, i.e., whether the layer closest to the substrate is a $\mathrm{TiO}_{2}$ or an $\mathrm{Al}_{2} \mathrm{O}_{3}$ layer. The other two samples comprise 10 alternating layers with double thickness $d=20 \mathrm{~nm}$, likewise with different layer ordering. Note that in the symbolic representation, where $H$ and $L$ refer to $10 \mathrm{~nm}$ thick titania and alumina layers, respectively, these four samples can be unambiguously denoted as $[H L H L]^{5},[L H L H]^{5},[H H L L]^{5}$, and $[L L H H]^{5}$ [see Fig. 1(c)]. 
If the EMA was valid, all four samples would be homogenized to the same $D=200 \mathrm{~nm}$ thick slab of anisotropic material with permittivity tensor components

$$
\epsilon_{\|}=\frac{1}{D} \sum_{j=1}^{N} d n_{j}^{2}, \quad \frac{1}{\epsilon_{\perp}}=\frac{1}{D} \sum_{j=1}^{N} d n_{j}^{-2},
$$

in the directions parallel and perpendicular to the layers, respectively. Here, $N$ is the number of layers and $n_{j}$ is the refractive index of the $j$ th layer $[13,14]$. Therefore, the EMA predicts that all samples feature identical reflection spectra. Thus, the difference in the reflectances between the samples signifies the breakdown of the EMA.

Quantitatively, this breakdown can be analyzed by determining the following two quantities:

$\left.\Delta R_{d} \equiv R\right|_{d=10 \mathrm{~nm}}-\left.R\right|_{d=20 \mathrm{~nm}},\left.\quad \Delta R_{o} \equiv R\right|_{H \ldots}-\left.R\right|_{L \ldots}$,

which reflect the sensitivity of the reflectance spectra towards layer thickness $d$ and layers ordering, respectively. Specifically for the fabricated samples, we can introduce

$$
\begin{aligned}
\Delta R_{d}^{(H)} & =R_{[H L H L]^{5}}-R_{[H H L L]^{5}}, \\
\Delta R_{d}^{(L)} & =R_{[L H L H]^{5}}-R_{[L L H H]^{5}} ; \\
\Delta R_{o}^{(20)} & =R_{[H H L L]^{5}}-R_{[L L H H]^{5}}, \\
\Delta R_{o}^{(10)} & =R_{[H L H L]^{5}}-R_{[L H L H]^{5}} .
\end{aligned}
$$

Figure 2 presents the calculated theoretical dependencies of $\Delta R_{d}$ and $\Delta R_{o}$ on the wavelength and angle of plane wave incidence for both polarizations of light (TE and TM). The calculations were performed by the standard transfer matrix approach as used in [9] with refractive indices of the materials either measured by spectroscopic ellipsometry
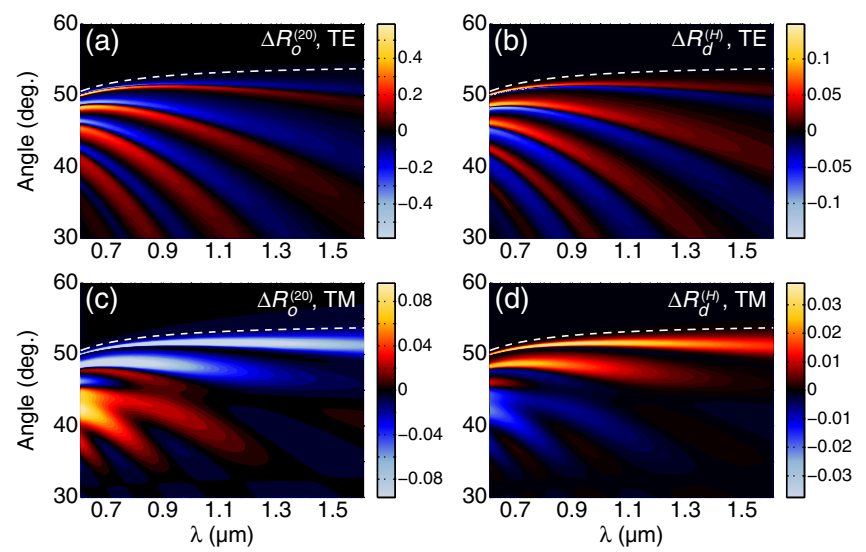

FIG. 2 (color online). Calculated reflectance differences (a) $\Delta R_{o}^{(20)}(\lambda, \theta)$, (b) $\Delta R_{d}^{(H)}(\lambda, \theta)$ for TE-polarized light at nearTIR angles of incidence; (c)-(d) Same as (a)-(b) but for TMpolarized light. The dashed line shows the spectral position of the TIR angle $\theta_{\text {TIR }}(\lambda)$ in the presence of material dispersion.
$\left(\mathrm{Al}_{2} \mathrm{O}_{3}, \mathrm{TiO}_{2}\right.$, and $\left.\mathrm{Si}_{3} \mathrm{~N}_{4}\right)$, or obtained from literature (silicon $[15,16]$ ) and manufacturer-provided data (zinc selenide [17]). Thus, dispersion in all materials is accurately taken into account.

In accordance with our earlier theoretical predictions for plane wave incidence [9], Fig. 2 shows a series of characteristic peaks in both $\Delta R_{d}$ and $\Delta R_{o}$ with the shape dependent on polarization. These EMA breakdown peaks occur at incidence angles $\theta$ below $\theta_{\mathrm{TIR}}(\lambda)$ (see the dashed lines in Fig. 2). The breakdown effect is generally more pronounced in the TE polarization and is more sensitive to the ordering of layers than to the variation of layers thicknesses. The values of $\Delta R$ are seen to decrease for larger wavelengths. However, the typical peak values of $|\Delta R|$ for the target range $\lambda=610-1610 \mathrm{~nm}$ are between 0.05 and 0.25 , and can even reach 0.5 , which is favorable for the measurements. Such high values of what would otherwise be a very weak effect are brought about by the resonator layer beneath the subwavelength structure [9].

To observe the EMA breakdown effect experimentally, we employed a modified Otto-Kretchmann configuration as illustrated in Fig. 1(a). A multilayer sample is placed in close proximity to a semicylindrical $\mathrm{ZnSe}$ prism, which is sufficiently high-index to achieve TIR at $\theta \sim 50^{\circ}$. The light source was a supercontinuum broadband laser (SuperK, NKT Photonics A/S, $\lambda=600-2500 \mathrm{~nm}$ ). Its collimated output beam was polarized by a double Glan-Thompson polarizer and focused at the ZnSe-sample interface, using a set of parabolic mirrors. The reflected beam was collected to a multimode fiber using another parabolic mirror and led into an optical spectrum analyzer (OSA, Yokogawa Electric Corp.) with the measuring range $\lambda=350-1750 \mathrm{~nm}$.

The sample was attached to the prism with a custommade holder tightened by a small-diameter screw. To minimize the air gap, which would have a dramatic influence on reflectance measurements according to the modeling results (especially for TM polarization), and to reduce the risk of dust trapping between the prism and the sample, the attachment of the sample was performed in the clean room. The quality of the attachment was monitored visually by controlling the appearance of the Newton rings around the location of the screw on the holder as the screw was tightened; see Figs. 3(b)-3(c). These rings originate from the interference between the back surface of the prism and the surface of the sample as the latter was pressed to the prism at the screw location, resulting in a variable air gap between the sample and the prism. The optical setup was aligned so that the incident beam was aimed at the innermost region of the ring pattern as shown in Figs. 3(b)-3(c).

Incident angle $\theta$ was varied by a rotating stage with precision $0.17^{\circ}$; another rotating stage was used to hold the collecting block (mirrors and fiber) for the reflected beam [Fig. 3(a)]. The focused incident beam was found to span a range $\phi$ of incident angles $\theta$. It was wavelength-dependent and estimated to vary from $\phi=1.3^{\circ}$ at $\lambda=600 \mathrm{~nm}$ to 
(a)

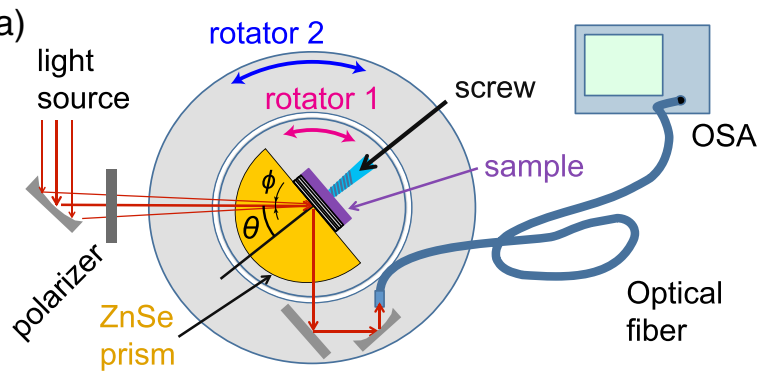

(b)

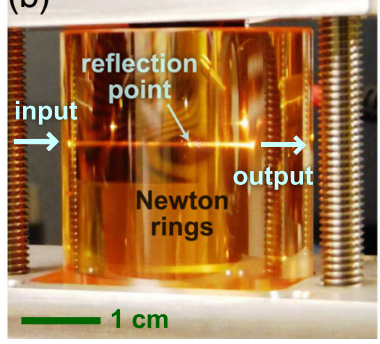

(c)

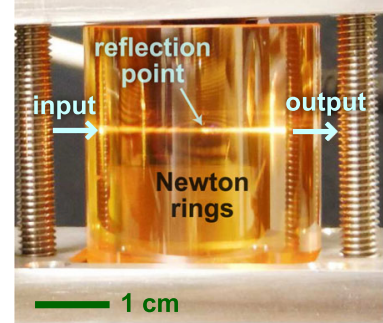

FIG. 3 (color online). (a) Schematics of the experimental setup for reflectance measurements. Photos of (b) $20 \mathrm{~nm}$ titania terminated sample $[L L H H]^{5}$ and (c) $10 \mathrm{~nm}$ alumina terminated sample $[H L H L]^{5}$ affixed to the $\mathrm{ZnSe}$ prism taken from the prism side and showing the appearance of the Newton rings, as well as the input and output light beams.

$\phi=3.9^{\circ}$ at $\lambda=2 \mu \mathrm{m}$. For each angle $\theta$, intensity spectra were obtained through averaging over 9 independent measurements. The measured spectra were normalized by a reference spectrum obtained by averaging 3 aboveTIR spectra $\left(\theta=53^{\circ}, 54^{\circ}, 55^{\circ}\right)$, since $R=1$ for $\theta>\theta_{\text {TIR }}$. The estimated wavelength-averaged error in the reflectance spectra was $4 \%$.

In order to compare with the experimental measurements, the calculated theoretical spectra were averaged over the wavelength-dependent angle interval $\phi$ corresponding to the beam divergence, as described above. Additionally, a mismatch in $\theta$ equal to $\Delta \theta=0.2^{\circ}$ (TE) and $\Delta \theta=0.3^{\circ}$ (TM) was introduced to compensate for setup misalignment. These values of $\Delta \theta$ were determined by minimizing the error between measured and experimental spectra in multivariate optimization (see the Supplemental Material [18]). The optimization additionally confirmed that there is no air gap between the prism and the thin-film sample, so an optical contact between them was achieved without the use of immersion liquid (impossible at such high refractive indices).

Both experimental and theoretical results for $\Delta R_{o}$ and $\Delta R_{d}$ in a variety of cases are shown in Fig. 4 for a range of $\theta$. We see that $\Delta R_{o}^{(20)}$ in the TE polarization [Fig. 4(a)] exhibits small-amplitude ripples across the spectrum for lower values of $\theta\left(20-30^{\circ}\right)$, in line with the expectation of Fig. 2(a). As $\theta$ approaches $\theta_{\mathrm{TIR}}$, the EMA breakdown becomes stronger, and $\Delta R$ reaches values around 0.4-0.5. Above $\theta_{\text {TIR }}, \Delta R$ vanishes since light undergoes TIR (a) $\Delta \boldsymbol{R}_{o}^{(20)}(\mathrm{TE})$

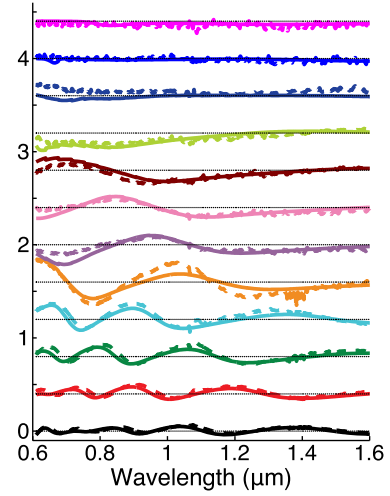

(c) $\Delta \boldsymbol{R}_{o}^{(10)}(\mathrm{TE})$

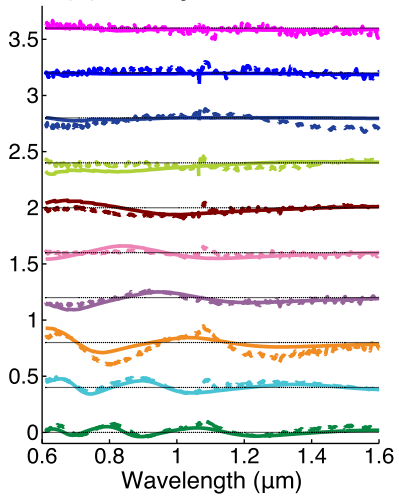

(b) $\Delta \boldsymbol{R}_{o}^{(20)}(\mathrm{TM}) \quad \theta$

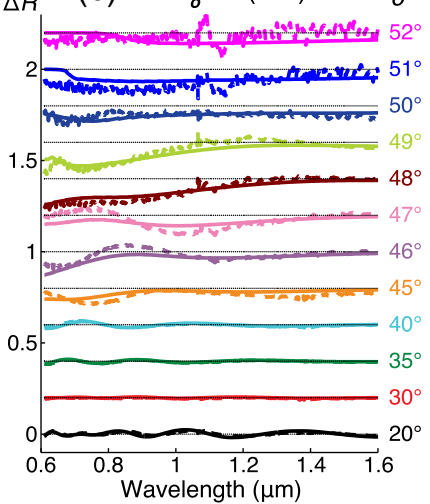

(d) $\Delta \boldsymbol{R}_{d}^{(L)}(\mathrm{TE})$

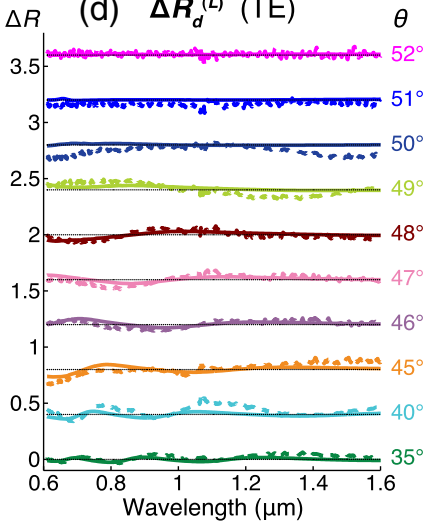

FIG. 4 (color online). Comparison between experimentally measured (dashed lines) and theoretically calculated (solid lines) reflectance differences: $\Delta R_{o}^{(20)}$ for (a) TE and (b) TM polarization; (c) $\Delta R_{o}^{(10)}$ and (d) $\Delta R_{d}^{(L)}$ for the TE polarization. The curves for different incident angles (marked on the right) are shifted vertically by 0.4 (TE) and 0.2 (TM) for the sake of convenience in comparison.

$(R=1)$ for both samples. Overall, the measured $\Delta R_{o}^{(20)}$ behaves very close to the theoretical predictions. Spectral disturbances around $1.06 \mu \mathrm{m}$ are artifacts arising from a very strong peak in the emission spectrum of the supercontinuum laser source around its pump laser wavelength (1064 nm).

For the corresponding $\Delta R_{o}^{(20)}$ in the TM polarization [see Fig. 4(b)], the reflectance differences have a lower amplitude and fewer characteristic spectral features than for the TE case. However, nonzero $\Delta R$ both in theory and in experiment is still apparent. Similar behavior as for $\Delta R_{o}^{(20)}$, albeit with proportionally smaller amplitudes, is observed for $\Delta R_{o}^{(10)}$ [Fig. 4(c)], as well as for $\Delta R_{d}^{(L)}$ [Fig. 4(d)]. There is still a good agreement between theory and experiment. The measured reflectance difference exceeds the experimental error (4\% average and 3\%-6\% depending on the wavelength) and reproduces most of the theoretically predicted spectral features. 
In summary, we have experimentally demonstrated the effect of the EMA breakdown for all-dielectric multilayers with deeply subwavelength layer thicknesses $(1 / 30$ to $1 / 160$ of the incident light wavelength). We have shown that the effect is present even for relatively thin structures, with the total thickness of $200 \mathrm{~nm}$, also smaller than the wavelength. The EMA breakdown manifests as the difference in the reflectance spectra of structures with different layer thickness (20 vs $10 \mathrm{~nm}$ ) as well as different layer ordering (whether the layer closer to the substrate is a highor a low-index layer), as shown in Fig. 1(c). The measured reflectance difference spectra, reaching values of around 0.5 , are in good agreement with theoretical transfer matrix calculations (Fig. 4).

Our results can be used in ellipsometry of multilayer structures, both to correct the existing ellipsometry models that rely on the EMA, and to devise new models specifically based on the measurement of the features related to the EMA breakdown.

As regards the applicability of the obtained results to sensing (using the high sensitivity of the EMA breakdown to the incident angle and the refractive index behind the multilayer [9]), we see that the spectral features in the reflectance spectra are not as sharp as theoretically predicted. This is primarily due to the use of the hemicylindrical prism together with a focused beam from a broadband light source, causing the light waves that reach the sample to have a finite range $\phi$ of incident angles $\theta$ and therefore smearing the spectral features. It is anticipated that adjusting the experimental setup to reduce this range of angles (e.g., by using a triangular prism or a light source with a collimated output) would lead to sharper spectral features in the reflectance difference spectra, making the device more suitable for high-sensitivity angle and refractive index measurements. Figure 2 can be regarded as the ultimate reference for sensing performance, showing the reflectance difference obtainable in an ideal experiment.

The authors acknowledge fruitful discussions with A. Novitsky. Partial support from the People Programme (Marie Curie Actions) of the EU 7th Framework Programme FP7-PEOPLE-2011-IIF under REA Grant Agreement No. 302009 (Project HyPHONE) is gratefully acknowledged. A. A. acknowledges support from the
Danish Council for Independent Research via the GraTer project (Contract No. 0602-02135B).

*sezh@fotonik.dtu.dk

†alav@fotonik.dtu.dk

[1] L. M. Brekhovskikh, Waves in Layered Media, 2nd ed. (Academic Press, New York, NY, 1980).

[2] M. Born and E. Wolf, Principles of Optics, 7th ed. (Cambridge University Press, Cambridge, England, 1999).

[3] P. Yeh, Optical Waves in Layered Media (Wiley, New York, 1988).

[4] H. A. Macleod, Thin-Film Optical Filters, Fourth Edition, Series in Optics and Optoelectronics (Taylor \& Francis, London, 2010).

[5] J. D. Joannopoulos, S. G. Johnson, J. N. Winn, and R. D. Meade, Photonic Crystals: Molding the Flow of Light, edition 2nd ed. (Princeton Unuversity Press, Princeton, NJ, 2008).

[6] D. Chigrin, A. Lavrinenko, D. Yarotsky, and S. Gaponenko, J. Lightwave Technol. 17, 2018 (1999).

[7] C. R. Simovski, J. Opt. 13, 013001 (2011).

[8] H. H. Sheinfux, I. Kaminer, Y. Plotnik, G. Bartal, and M. Segev, Phys. Rev. Lett. 113, 243901 (2014).

[9] A. Andryieuski, A. V. Lavrinenko, and S. V. Zhukovsky, Nanotechnology 26, 184001 (2015).

[10] N. Pinna and M. Knez, Atomic Layer Deposition of Nanostructured Materials (Wiley-VCH Verlag \& Co. KGaA, New York, 2012).

[11] W.-J. Lee and M.-H. Hon, J. Phys. Chem. C 114, 6917 (2010).

[12] D. Mitchell, D. Attard, K. Finnie, G. Triani, C. Barbé, C. Depagne, and J. Barlett, Appl. Surf. Sci. 243, 265 (2005).

[13] V. M. Agranovich, Solid State Commun. 78, 747 (1991).

[14] A. V. Lavrinenko and V. V. Zhilko, Microwave Opt. Technol. Lett. 15, 54 (1997).

[15] G. Vuye, S. Fisson, V. N. Van, Y. Wang, J. Rivory, and F. Abelès, Thin Solid Films 233, 166 (1993).

[16] H. Li, J. Phys. Chem. Ref. Data 9, 561 (1980).

[17] Crystran 1td., http://www.crystran.co.uk/, accessed: 201506-11.

[18] See Supplemental Material at http://link.aps.org/ supplemental/10.1103/PhysRevLett.115.177402 for the comparison of theoretical and experimental reflectance spectra, as well as for the description of optimization procedure used for the comparison. 